\title{
Laser Patterning of smart Nanomaterials for Reel-to-Reel production of Organic Photovoltaic (OPV) Devices
}

\author{
C. Moorhouse ${ }^{1}$, D. Karnakis ${ }^{1}$, C. Kapnopoulos ${ }^{2}$, A. Laskarakis ${ }^{2,3}$, E. Mekeridis ${ }^{4}$, S. Logothetidis ${ }^{2}$ \\ 1 Oxford Lasers, Unit 8 Moorbrook Park, Didcot, OX11 7HP, UK \\ 2 Lab for Thin Films, Nanosystems \& Nanometrology (LTFN), Aristotle University of Thessaloniki, 54124, Thessaloniki Greece \\ 3 Department of Materials Science and Engineering, University of Ioannina, Ioannina, Greece \\ 4 Organic Electronic Technologies P.C. (OET), Antoni Tritsi 21B, 57001 Thessaloniki, Greece
}

Main author email address: colin.moorhouse@oxfordlasers.com

\begin{abstract}
Organic photovoltaic (OPV) devices are attracting increasing commercial interest due to their low weight/size and flexibility, which allows them to be used on the exterior windows of cars, buildings, backpacks \& clothing. The direct write and non-contact nature of laser patterning is highly desirable for the P1, P2 \& P3 serial interconnection and isolation structures, as it is compatible with integration in sheet-to-sheet and reel-to-reel production lines. However, detrimental effects such as layer edge delamination or laser debris re-deposition can effect OPV performance and represent real technological problems hindering the adoption of laser schemes to production lines. Since, OPVs contain highly sensitive, thin $(<0.2 \mu \mathrm{m})$ layers of novel organic \& inorganic materials, the reduced thermal effects of sub-nanosecond and other ultrafast lasers along with intelligent beam delivery schemes are key to produce selective removal of individual thin film layers. OPV device performance was analyzed to identify the P1 laser scribing parameters and conditions required to produce working organic solar cells.
\end{abstract}

DOI: $10.2961 / \mathrm{jlmn} .2015 .02 .0017$

Keywords: ultrafast lasers, organic solar cells, beam shaping, reel-to-reel manufacturing, P1, P2, P3

\section{Introduction}

Organic photovoltaic (OPV) devices are attracting interest for use in several consumer applications, ranging from automotive to buildings with OPVs incorporated into windows, as a low weight/cost supply of electricity. One of the cutting-edge research activities includes the Smartonics project that concentrates on the development of smart nanomaterials (including thin film layers) and process technologies in order to integrate these in novel pilot lines for the fabrication of novel OPV devices with high efficiency and lifetime [1]. The key to keeping cost levels down is the development of reel-to-reel production lines with the different layers printed and patterned on plastic substrates. One way to improve OPV device performance is to take advantage of the plasmonic effect to boost the conversion efficiency [2] and more details on the device structures and manufacturing processes are outlined in reference [3].

Laser scribing is particularly attractive for OPV production lines compared to competitive technologies such as photolithography as it is a single step, direct write and vacuum-free process. Laser scribing also allows a reduction of the so called "dead area" of OPVs by tightly spacing the scribed interconnection lines and so utilising larger cell area for current generation as compared to current printing techniques [4]. Furthermore, the use of laser scribing allows for greater flexibility to use both coating and printing techniques as needed in OPV production lines. The OPV devices built in this study are large area devices $(120 \times 200 \mathrm{~mm})$ fabricated on flexible polyethylene terephthalate (PET) substrates for a sheet-to-sheet pilot line under ambient atmosphere, as opposed to vacuum deposition for other OPV devices [5]. There has been recent interest in laser scribing for OPV devices [4, 5], however, device structures and materials are still in their infancy. This report summarizes laser scribing of novel OPV device materials, while also outlining methods for laser scribing to meet the demands of reel-to-reel production lines. The OPVs used for this study have an active area of $6.74 \mathrm{~cm}^{2}$ and are manufactured on flexible PET substrates as opposed to glass [4] and the production line is under ambient atmosphere and not under vacuum as in other studies [5].

Laser patterning steps occur after deposition of each of the transparent electrode, active material and primary electrode layers. Typical stack layers for the OPV devices studied in this report and the P1, P2 and P3 laser scribes required are shown in Figure 1. P1 involves patterning of the transparent electrode, P2 removal of the active material down to the transparent electrode back contact and P3 is the patterning of the primary metal electrode. 

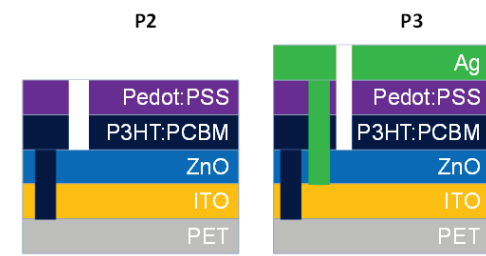

Figure 1 P1, P \& P3 laser scribes for OPV devices.

To prevent short circuits or shunts in the final devices, very low delamination and scribe edge burring less than $100 \mathrm{~nm}$ is required. Ultrafast lasers can be very attractive for selective removal of thin film organic device layers $[6,7]$ as in many instances the laser pulse duration is significantly shorter than the thermal and/or acoustic response times of the material and that presents a unique advantage. In such cases, a spallation driven mechanism can result in the intact layer removal at relatively low temperature, well below the material melt point. The selective laser removal process is shown schematically in Figure 2 where (a) a focused laser beam is incident on a thin film layer, (b) the light is absorbed within the layer and at the interface of the thin film, (c) forming a shock wave which expands outwards and (d) spallating the 'cold' thin film layer typically in solid flakes with minimal effect on the surrounding materials. For these trials, the laser beam is always incident from the coated side as indicated in Figure 2.
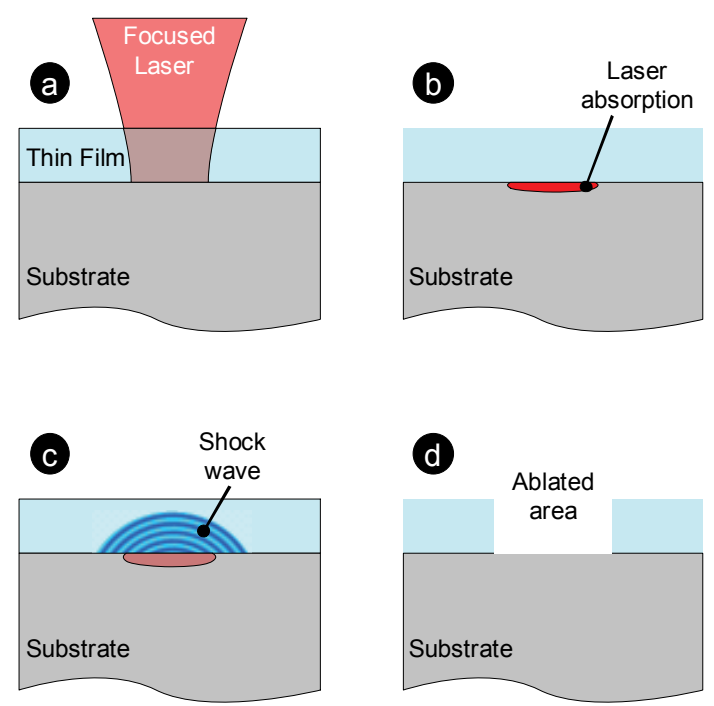

Figure 2 Selective laser removal process using ultrafast laser pulses.

\section{P1 Laser Scribing}

The transparent electrode consists of indium tin oxide (ITO), which can be readily removed from PET substrates using ultrafast lasers. For a laser process, the relevant wavelength for P1 scribing is chosen by considering Table 1, which tabulates the optical properties of ITO and PET for the common Diode Pumped Solid State (DPSS) laser wavelengths where $n$ is the real part of the refractive index, $k$, the imaginary part (extinction coeffienct), $\lambda$ wavelength and $\alpha=4 \pi \kappa / \lambda$, the estimated linear optical absorption coefficient. In the infrared, PET has low optical absorption (low $\alpha$ ), while ITO absorbs moderately better, so this wavelength was chosen for P1 scribing to avoid damaging the flexible substrate.

\section{Table 1 Optical properties of ITO and PET.}

\begin{tabular}{|c|c|c|c|}
\hline \multicolumn{1}{l}{ ITO } & \multicolumn{3}{l|}{} \\
\hline $\boldsymbol{\lambda}(\mathbf{n m})$ & $\mathbf{n}$ & $\mathbf{k}$ & $\boldsymbol{\alpha}\left(\mathbf{c m}^{-\mathbf{1}} \mathbf{)}\right.$ \\
\hline 1064 & 1.8 & 0.07 & $8.3 \mathrm{E}+03$ \\
\hline 532 & 2.1 & 0.1 & $2.4 \mathrm{E}+04$ \\
\hline 355 & 2.3 & 0.23 & $8.1 \mathrm{E}+04$ \\
\hline 266 & 2 & 0.6 & $2.8 \mathrm{E}+05$ \\
\hline PET & & & \\
\hline $\boldsymbol{\lambda}(\mathbf{n m})$ & $\mathbf{n}$ & $\mathbf{k}$ & $\boldsymbol{\alpha}\left(\mathbf{c m}^{-1} \mathbf{)}\right.$ \\
\hline 1064 & 1.75 & 0.04 & 4724 \\
\hline 532 & 1.82 & 1.38 & 325970 \\
\hline 355 & 1.87 & 1.48 & 523894 \\
\hline 266 & 1.6 & 1.8 & 850356 \\
\hline
\end{tabular}

The P1 laser scribes were tested for electrical isolation with a digital voltmeter with resistance $>10 \mathrm{M} \Omega$ measured across the scribes. The main issue was film delamination and flaking at the scribed edges. Figure 3 shows a comparison of individually optimized P1 scribes using different laser parameters with (a) picosecond and (b) femtosecond laser pulse overlaps of $17 \%$ and $25 \%$ respectively. For the picosecond laser scribes a focussed spot size of $24 \mu \mathrm{m}$ (at $1 / \mathrm{e}^{2}$ of max.intensity), pulse energy of $4.2 \mu \mathrm{J}$, repetition rate of $1 \mathrm{kHz}$ were used at a scribing speed of $15 \mathrm{~mm} / \mathrm{s}$. The femtosecond laser scribe was produced using a smaller focused spot size of $12 \mu \mathrm{m}$, lower pulse energy of $0.9 \mu \mathrm{J}$, repetition rate of $2 \mathrm{kHz}$ at a scribe speed of $15 \mathrm{~mm} / \mathrm{s}$. Overall, the picosecond laser scribes show more delamination, which is visible as the white areas on the ablated edges in Figure 3(a).

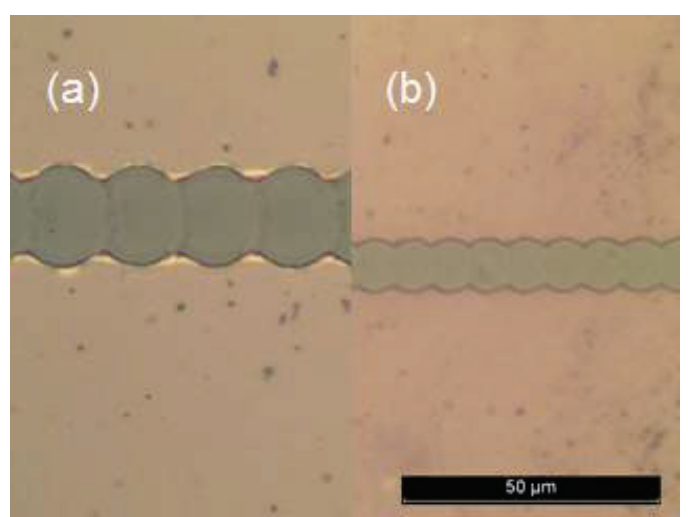

Figure 3 Comparison of P1 scribes with infrared (a) picosecond and (b) femtosecond lasers.

\subsection{Effect of pulse duration on P1 scribing}


The impact of the laser pulse duration on laser scribing was further investigated by measuring the variation in the measured laser ablation threshold, $F_{T h}$, between $310 \mathrm{fs}$ and $10 \mathrm{ps}$ at single pulse ablation [8, 9]. The plot of $F_{T h}$ versus pulse duration in Figure 4 shows the $F_{T h}$ remains roughly constant for pulse durations below 2 ps. Hence, a laser pulse duration of less than $2 \mathrm{ps}$ would not provide any additional benefits in terms of minimising ablation energy requirements and is taken to be optimum for P1 laser scribing in this work. Further investigations were not conducted regarding potential qualitative removal mechanism benefits.

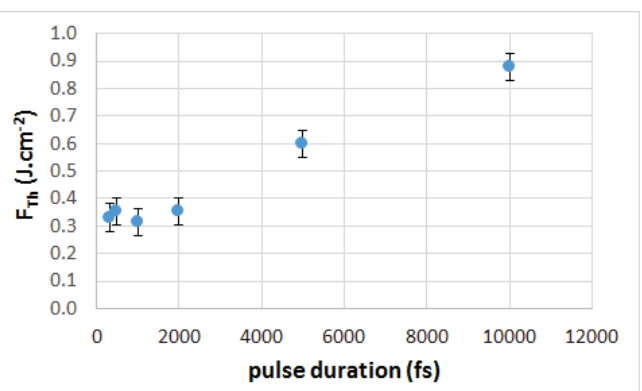

Figure 4 Effect of pulse duration on laser ablation threshold, $F_{T h}$, for P1 scribing.

The influence of focused spot size on film delamination was briefly investigated using SEM and confocal microscopy. Delamination being defined as the vertical extent of the raised scribed edges due to peeling of the thin film layer. The delamination was found to depend strongly on focused spot size with the lowest delamination obtained using spot sizes of less than $<25 \mu \mathrm{m}$. For example, in Figure 3(a) the delamination for 310fs laser pulses was $16 \pm 16 \mathrm{~nm}$ compared to $34 \pm 23 \mathrm{~nm}$ for 10ps pulses in Figure 3(b).

\subsection{Beam profile shaping for P1 scribing}

It has been shown that the use of smaller focused spot size improves the edge quality and reduces delamination. However, the use of a smaller spot size requires higher numerical aperture optics with shorter depth of focus, which makes maintaining the same fluence level across a moving reel-to-reel line more difficult to achieve. One alternative is to use a tophat intensity beam profile that exhibits a steeper intensity gradient near the edges than a Gaussian beam. The aperture mask imaging setup outlined in Figure 5 was implemented to produce a tophat beam profile at the workpiece. Using a $1 \mathrm{~mm}$ square aperture, a $40 \times 40 \mu \mathrm{m}$ square beam profile was imaged onto the workpiece, using a translation stage to move the laser beam relative to the substrate.

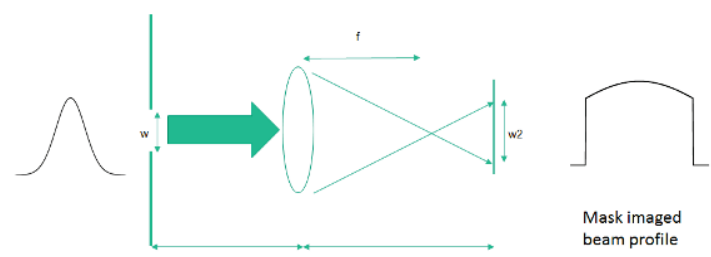

Figure 5 Mask imaging setup to produce a straight edge beam profile at the workpiece.

The scanning electron microscope (SEM) image in Figure 6 shows a P1 scribe made using aperture mask imaging with a $310 \mathrm{fs}$ laser pulse energy of $7.2 \mu \mathrm{J}$ and pulse overlap of $38 \%$ for a $40 \times 40 \mu \mathrm{m}$ square beam profile. It is clear that edge scalloping is minimised comparing to Fig. 3 (focused Gaussian beam). The delamination height at the edges was measured by confocal microscope to be $42 \pm 26 \mathrm{~nm}$, which is not quite as low as that obtained with a $12 \mu \mathrm{m}$ focused Gaussian beam $(16 \pm 16 \mathrm{~nm})$. Also, a post-process clean is required in this case to remove the micron scale flakes on the scribe edges visible in Figure 6 possibly emanating from a slight misalignment of the mask axis to the $\mathrm{XY}$ table motion axis. These flakes are not present for the Gaussian beam laser scribe in Figure 3(b), so for device trials, the P1 laser scribes were made with a Gaussian beam shape.

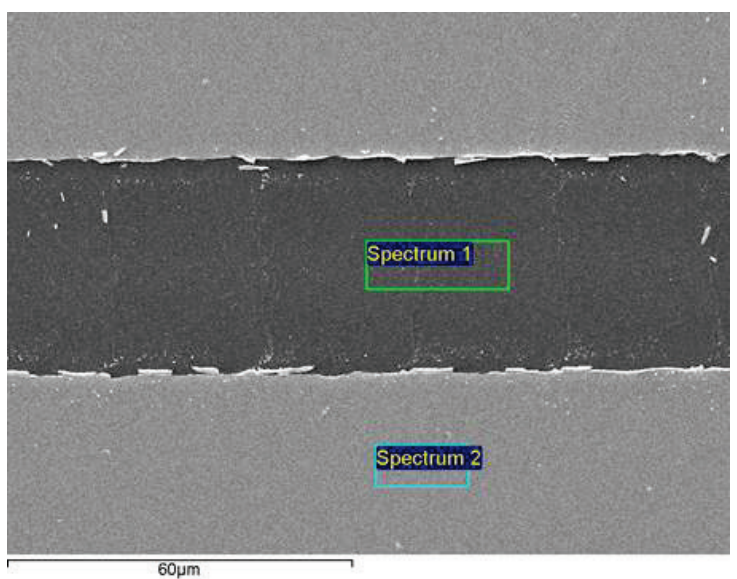

Figure 6 SEM image of ITO scribing using a mask imaged square fs laser beam.

\subsection{OPV devices with P1 laser scribes}

PET sheet-to-sheet OPV devices were fabricated containing P1 laser scribes using an industrial grade DPSS infrared 10ps laser, which is close to the 2 ps optimum outlined in Figure 4 . A postprocess ultrasound bath was used to clean the scribe edges after laser scribing with a comparison of the laser scribes before and after ultrasound cleaning shown in Figure 7. The focused spot size was $\sim 30 \mu \mathrm{m}$ and optimum scribes in Figure 7 (a) were obtained using a pulse energy of $17 \mu \mathrm{J}$ and pulse-to-pulse spacing of $20 \mu \mathrm{m}$. Confocal microscope measurements of the edge delamination showed the cleaning process improved the raised 
edge delamination from $100 \mathrm{~nm}$ height before cleaning to $20 \mathrm{~nm}$ after ultrasound cleaning. These P1 laser scribes resulted in a conversion efficiency of $0.66 \%$, which is an encouraging initial result for this project. This result highlights the importance of laser related debris (flakes, redeposited particles) which is an ongoing area of research within this project.

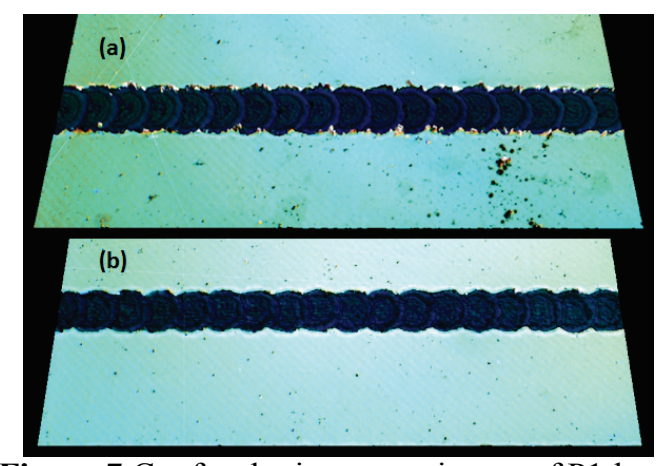

Figure 7 Confocal microscope image of $\mathrm{P} 1$ laser scribes made using an infrared, 10ps laser (a) before and (b) after ultrasound cleaning.

\section{P2 Laser Scribes}

The P2 laser scribe removes the active material, P3HT:PCBM and PEDOT:PSS (see Figure 1) from the ITO and the optical properties of the active material are shown in Table 2 P3HT:PCBM absorbs strongly, in the visible region of the spectrum, so it is anticipated that second harmonic upconverted, ultrafast lasers (SHG) emitting around $532 \mathrm{~nm}$ would offer the best option to obtain selective removal of the active material. The PEDOT:PSS layer above the active layer is largely transparent to both 532 and $1064 \mathrm{~nm}$, however, the strong absorption in the visible for P3HT:PCBM should allow the PEDOT:PSS layer to be removed cleanly as well.

\section{Table 2 Optical properties of P3HT:PCBM and} PEDOT:PSS.

\begin{tabular}{|c|c|c|c|c|c|c|c|}
\hline \multicolumn{8}{|c|}{ Р3НТ:РСВМ } \\
\hline$\lambda(\mathrm{nm})$ & \multicolumn{2}{|l|}{$\mathbf{n}$} & \multicolumn{2}{|l|}{ k } & \multicolumn{2}{|c|}{$\alpha\left(\mathrm{cm}^{-1}\right)$} & $1 / \alpha(\mu \mathrm{m})$ \\
\hline 1064 & \multicolumn{2}{|c|}{1.85} & \multicolumn{2}{|r|}{0} & \multicolumn{2}{|c|}{$0.0 \mathrm{E}+00$} & 0.000 \\
\hline 532 & \multicolumn{2}{|c|}{2.1} & \multicolumn{2}{|c|}{0.77} & \multicolumn{2}{|c|}{$1.8 \mathrm{E}+05$} & 0.055 \\
\hline 355 & \multicolumn{2}{|c|}{1.55} & \multicolumn{2}{|c|}{0.2} & \multicolumn{2}{|c|}{$7.1 \mathrm{E}+04$} & 0.141 \\
\hline 266 & \multicolumn{2}{|c|}{1.48} & & 0.2 & \multicolumn{2}{|c|}{$9.4 \mathrm{E}+04$} & 0.106 \\
\hline \multicolumn{8}{|c|}{ Pedot:PSS } \\
\hline$\lambda(\mathrm{nm})$ & \multicolumn{2}{|l|}{$\mathbf{n}$} & \multicolumn{3}{|c|}{$\alpha\left(\mathrm{cm}^{-1}\right)$} & $1 / \alpha(\mu \mathrm{m})$ & $\mathbf{R}(\%)$ \\
\hline 1064 & 1.55 & \multicolumn{2}{|r|}{0} & \multicolumn{2}{|c|}{$0.0 \mathrm{E}+00$} & 0.000 & $4.65 \%$ \\
\hline 532 & 1.56 & \multicolumn{2}{|r|}{0} & \multicolumn{2}{|c|}{$0.0 \mathrm{E}+00$} & 0.000 & $4.79 \%$ \\
\hline 355 & 1.58 & \multicolumn{2}{|r|}{0} & \multicolumn{2}{|c|}{$0.0 \mathrm{E}+00$} & 0.000 & $5.05 \%$ \\
\hline 266 & 1.7 & \multicolumn{2}{|c|}{1.4} & \multicolumn{2}{|c|}{$6.6 \mathrm{E}+05$} & 0.015 & $26.49 \%$ \\
\hline
\end{tabular}

Many different laser combinations of wavelength and pulse duration were tested including: $532 \mathrm{~nm} \mathrm{ns,}$ $532 \mathrm{~nm}$ sub-ns, infrared ps and fs lasers, all of which did not show sufficient selectivity to make successful P2 scribes. Visible emitting SHG ultrafast lasers were found to be most suitable. Figure 8 shows P2 scribes made using a 532nm,
$10 \mathrm{ps}$ laser focused to a $14 \mu \mathrm{m}$ spot size with repetition rate of $1 \mathrm{MHz}$, pulse energy $0.38 \mu \mathrm{J}$ and scribe speed of $2.5 \mathrm{~m} / \mathrm{s}$. Similar scribes are shown in Figure 9 for a $515 \mathrm{~nm} 310 \mathrm{fs}$ laser focused to a spot size of $\sim 13 \mu \mathrm{m}$, pulse energy $0.18 \mu \mathrm{J}$, repetition rate of $1 \mathrm{MHz}$ and scribe speed of $6 \mathrm{~m} / \mathrm{s}$. The Energy Dispersive X-Ray (EDX) spectrum \#1 in Figure 8 and spectrum \#2 in Figure 10, are taken in the centre of the scribe regions and both show strong indium (In) and zinc (Zn) peaks indicating that the back contact layer is exposed. The lack of sulphur (S) peaks in the scribed region indicates that the active material has been completely removed. For both lasers, the ITO is acting as an etch stop layer enabling a scribe structure like that in Figure 1. It should be noted that organic contamination was observed on several sample surfaces prior to laser irradiation (shown in Figure 8 ), the source of which was unknown. This was not related to the laser patterning and was frequently observed on other test sample materials, most likely an artefact of their printed deposition. A cleaning post-process step is not possible after P2 and P3 scribing due to the sensitivity of the thin film layers to abrasion. Also, since the back contact is a conductive layer it was not possible to make electrical isolation measurements to verify if the layers are completely removed.
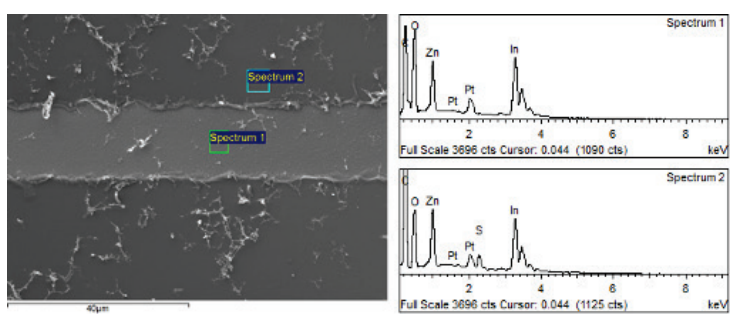

Figure 8 SEM image of P2 scribe made with a $532 \mathrm{~nm}, 10 \mathrm{ps}$ laser and EDX spectra inside and outside the scribed region.

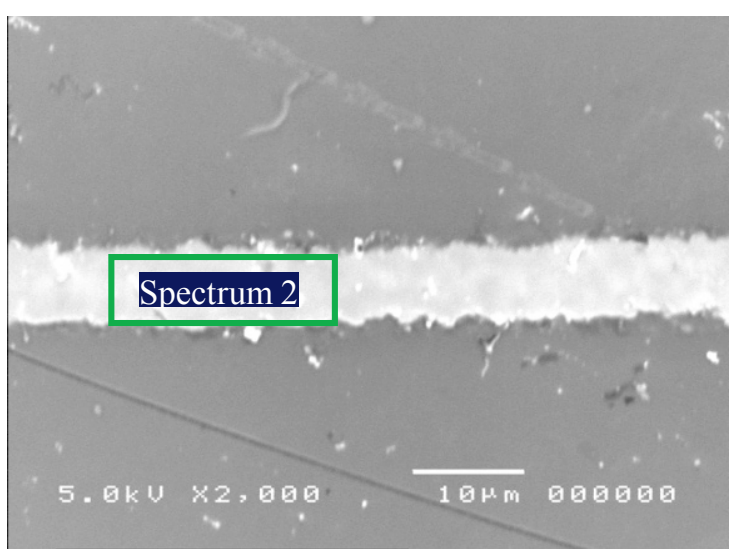

Figure 9 SEM image of P2 scribe using $515 \mathrm{~nm}$ fs laser. 


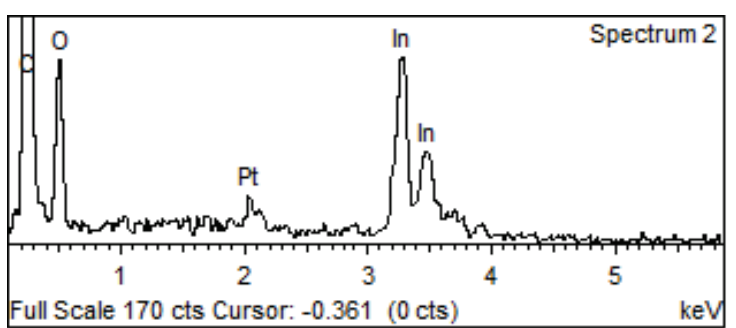

Figure 10 EDX analysis of the laser scribed region in Figure 9 showing the ITO layer is present.

A similar mask imaging setup to Figure 5 was used with a $\lambda=515 \mathrm{~nm}, 310 \mathrm{fs}$ laser to produce a $\sim 35 \times 35 \mu \mathrm{m}$ square beam at the workpiece and a $30 \mu \mathrm{m}$ wide P2 scribe width is shown in Figure 11. Again, the ITO acts effectively as an etch stop and a P2 scribe has been produced with little or no visible debris and really straight edges. The delamination at the scribe edges was measured using SEM and confocal microscope images to be $68 \mathrm{~nm}$, which was slightly greater than the $44 \mathrm{~nm}$ delamination obtained using $310 \mathrm{fs}$ and 10ps lasers with a Gaussian beam focused to a spot size of $12 \mu \mathrm{m}$.

The effect of pulse duration was again estimated by measuring the laser ablation threshold, $F_{T h}$ values for the 10ps and 310fs laser (as in section 2.1). However, it was not possible to vary the pulse duration for the second harmonic of the femtosecond laser, so only two $F_{T h}$, values were

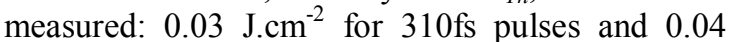

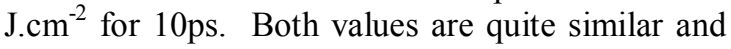
represent a very low fluence requirement given the sensitivity of the layers. Furthermore, the delamination/burr of the P2 scribe edges (as measured by SEM and confocal microscope analysis) was found to be approximately $100 \mathrm{~nm}$ for both $310 \mathrm{fs}$ and $10 \mathrm{ps}$ lasers. Hence, for actual test device trials, OPV devices with P2 scribes made using a $\lambda=532 \mathrm{~nm}, 10 \mathrm{ps}$ laser will be implemented in the near future.

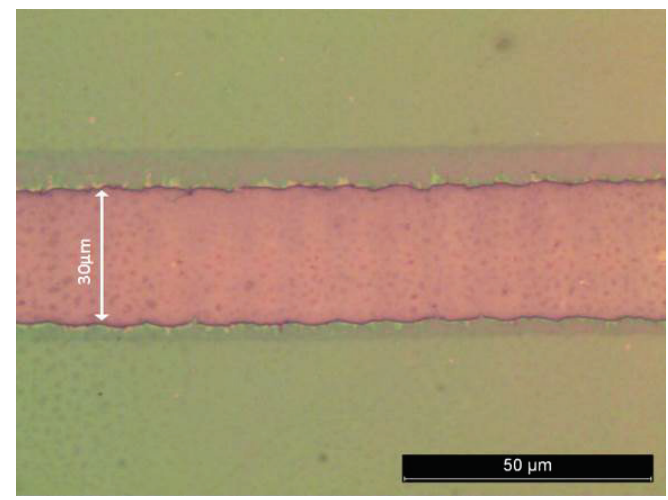

Figure $11 \mathrm{P} 2$ scribe produced using a $515 \mathrm{~nm}$, fs laser with a mask imaged square beam profile of $35 \times 35 \mu \mathrm{m}$.

\section{P3 Laser Scribes}

There are two possible P3 scribe configurations outlined in Figure 12: (a) to remove the Ag contact layer and PEDOT:PSS to expose the active material or (b) remove the Ag, PEDOT:PSS and active material exposing the rear contact layer. Both configurations were investigated and the best working one will be confirmed by testing OPV devices. From the optical properties in Table 2, both the PEDOT:PSS and P3HT:PCBM are highly transparent to infrared wavelengths, hence, selective removal of both the silver contact and PEDOT:PSS layers from the active material is anticipated to be difficult using infrared lasers. (a)

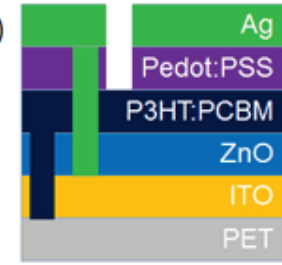

(b)

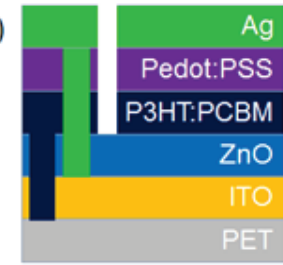

Figure 12 P3 scribe configurations possibilities.

As an example, a P3 scribe with a possible exposed active region like that in Figure 12(a) is shown in Figure 13. 10ps infrared laser pulses were used with a focused spot size of $40 \mu \mathrm{m}$ at a pulse energy of $4.2 \mu \mathrm{J}$, rep. rate of $300 \mathrm{kHz}$ and scribe speed of $4.5 \mathrm{~m} / \mathrm{s}$. Both the PEDOT:PSS and P3HT:PCBM layers contain sulphur, which complicates the qualitative EDX analysis. The EDX spectra \#1 and \#2 in Figure 14 from the central scribed area show some sulphur $\mathrm{S}$ content, which indicates the presence of PEDOT:PSS or P3HT:PCBM traces . The EDX spectrum \#3, closer to the scribe edge shows a slightly stronger $\mathrm{S}$ peak, which indicates the presence of PEDOT:PSS (especially when compared to other SEM images/EDX spectra, not shown here ). Whilst the qualification of such thin layer removal selectivity is clearly difficult and inconclusive, further practical testing is ongoing to confirm if the P3 scribe in Figure 13 is viable for OPV device fabrication.

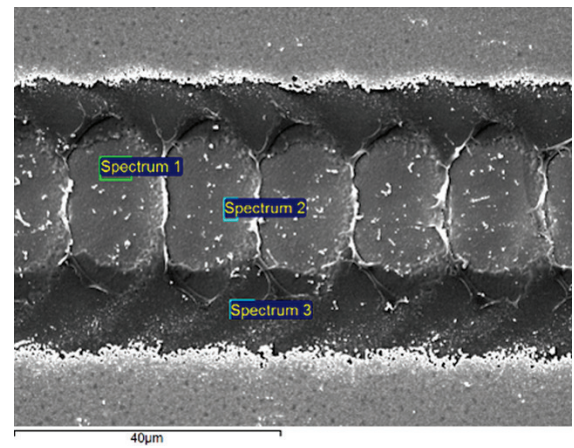

Figure 13 SEM image of a $1064 \mathrm{~nm}$ ps laser P3 scribe and EDX spectra of the inner scribe region. 


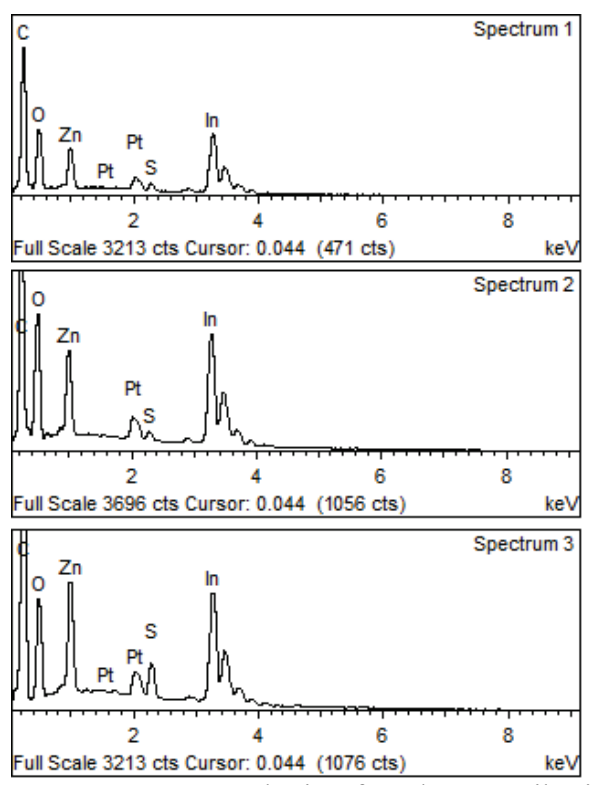

Figure 14 EDX analysis of P3 laser scribe in Figure 13.

The strong absorption of the active material (see Table 2) was found to prevent selective removal of the active material using visible (515 and 532nm) wavelength ultrafast lasers. However, obtaining the P3 scribe profile in Figure 12(b) is less complicated and P3 laser scribes were made with both infrared and SHG ps lasers. The SEM image in Figure 15 shows a P3 scribe removing all layers, exposing the rear contact $\mathrm{ZnO} / \mathrm{ITO}$ layer using an infrared ps laser at a fluence of $0.3 \mathrm{~J} . \mathrm{cm}^{-2}$ and scribe speed of $4 \mathrm{~m} / \mathrm{s}$ for a laser repetition rate of $200 \mathrm{kHz}$ and focused spot size of $80 \mu \mathrm{m}$. The EDX spectrum \#2 show no sulphur peaks (indicating that the PEDOT:PSS and P3HT:PCBM are completely removed). The presence of $\mathrm{Zn}$ and In peaks in the scribe region indicates that the active material has been completely removed to produce a P3 scribe like that in Figure 1.

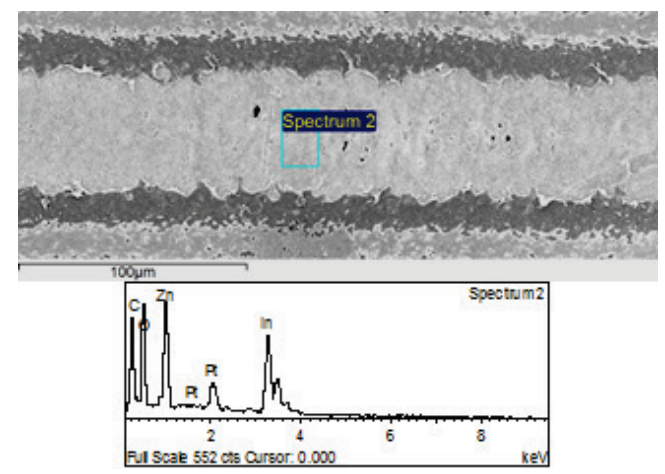

Figure 15 SEM image of a $1064 \mathrm{~nm}$ ps laser scribe exposing the back contact $\mathrm{ZnO} / \mathrm{ITO}$ layer, which is confirmed by the EDX spectrum.

Using 532nm picosecond laser pulses focused to $\sim 40 \mu \mathrm{m}$ spot size, the P3 scribe shown in Figure 16, which is similar to the scribe profile in Figure 12(b).
The EDX spectrum \#1 in Figure 16 in the central scribe area has strong $\mathrm{Zn}$ and In peaks, indicating the $\mathrm{ZnO} / \mathrm{ITO}$ rear contact layer has been exposed. Using these laser conditions, a scribe speed of $1 \mathrm{~m} / \mathrm{s}$ was possible at a laser repetition rate of $200 \mathrm{kHz}$. Also, a laser fluence of $0.14 \mathrm{~J} . \mathrm{cm}^{-2}$ was used, which is approximately half the fluence requirement for infrared laser pulses. Furthermore, the laser scribe profile is cleaner in appearance than that the produced by infrared laser pulses in Figure 15 (despite the misleading presence of some unknown organic contamination in and around the laser scribe, which was not related to laser treatment).

The extra complexity of the multi-layered P3 material resulted in greater delamination (compared to P1 and P2) of the order 100-200nm for all these scribes. The P3 scribes in Figure 13, Figure 15 and Figure 16 show different options for P3 scribing within OPV devices and the next phase of the project will investigate the influence of these scribes on device performance.

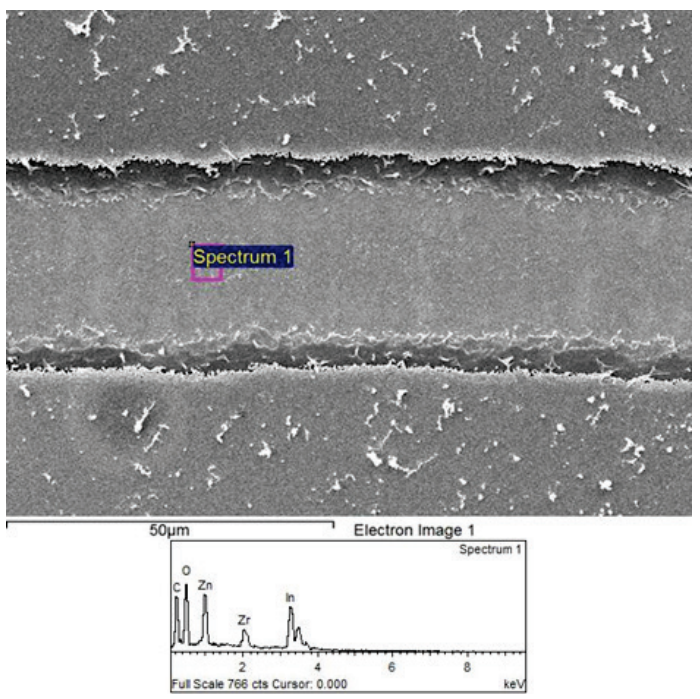

Figure $16532 \mathrm{~nm}$ ps laser P3 scribe exposing the back contact $\mathrm{ZnO} / \mathrm{ITO}$ layer, which is confirmed by the EDX analysis of the scribed region.

Overall, both the P2 and P3 laser processes exhibit low fluence requirements of less than $<0.5 \mathrm{~J} . \mathrm{cm}^{-2}$, so in order to obtain uniform laser scribing across the full OPV device and from cell to cell, it is essential to ensure homogeneously coated device layers and DPSS lasers operating with very good pulse-to-pulse output stability of better than $2 \%$.

\section{Conclusions}

A number of different laser types have been tested for P1, P2 and P3 scribing for an OPV pilot production line. For P1 scribes, infrared picosecond lasers were found to produce OPV device efficiencies of $0.66 \%$ and efforts are ongoing to improve the laser and non-laser 
processes further to increase this value. Green ps lasers can be used to produce P2 and P3 scribes and these will be incorporated into devices in the near future to find the impact on the OPV device performance. The low fluence requirement means ultrafast lasers with highly stable performance will be key to achieving consistent laser scribes.

\section{References}

1. $\quad$ EU FP7 NMP.2012.1.4-1 Large Scale

Project Smartonics (http://www.smartonics.eu).

2. Beliatis, M. J., Henley, S. J., Han, S., Gandhi, K., Adikaari, A. A. D. T., Stratakis, E., Kymakis, E., Ravi, S., Silva, P. , "Organic solar cells with plasmonic layers formed by laser nanofabrication." Physical Chemistry Chemical Physics 15(21), pp. 8237-8244, 2013.

$3 . \quad$ Koidis C., Logothetidis, S., Kassavetis, S., Kapnopoulos, C., Karagiannidis, P.G., Georgiou, D., Laskarakis, A., "Effect of process parameters on the morphology and nanostructure of roll-to-roll printed P3HT:PCBM thin films for organic photovoltaics," Solar Energy Materials \& Solar Cells 112, pp. 36-46, 2013.

4. Li, N., P. Kubis, Foberich, K., Ameri, T., Krebs, F. C., Brabec, C. J., "Towards large-scale production of solution-processed organic tandem modules based on ternary composites: Design of the intermediate layer, device optimization and laser based module processing." Solar Energy Materials \& Solar Cells 120, pp. 701-708, 2014.

5. Gebhardt, M., Hanel, Jens, Allenstein, F., Scholz, C., Maurice, C., "Laser Structuring of Flexible Organic Solar Cells." Laser Technik 10(1), pp. 25-28, 2013.

6. Karnakis, D. M., Kearsley, A. Knowles M. R. H., "Ultrafast Laser Patterning of OLEDs on Flexible Substrate for Solid-state Lighting," Journal of Laser Micro/Nanoengineering, vol. 4(3), pp. 218-223, 2009.

7. Moorhouse, C. Karnakis, D. M., Mandamparambil, R., "Patterning of Organic, Electronic Devices Using a High Average Power Picosecond Laser," Proceedings ICALEO, Paper \#M901, 2011.

8. Liu, J. M., "Simple technique for measurment of pulsed Gaussian-beam spot sizes." Optics Letters 7(5), pp. 196-198, 1982.

9. Lednev, V. N., Pershin, S. M., Obraztsova, E. D., Kudryashov, S. I., Bunkin, A. F. , "Singleshot and single-spot measurement of laser ablation threshold for carbon nanotubes." Journal of Physics D: Applied Physics, 46(5): 052002, 2013.

\section{Acknowledgements}

The research leading to these results has received funding from the European Union Seventh Framework Programme (NMP.2012.1.4-1 Project Smartonics) under Grant Agreement number 310229. We would like to thank the University of
Oxford for use of their advanced metrology facility at Begbroke Science Park (high resolution SEM and EDX).

(Received: July 29, 2014, Accepted: March 24, 2015) 